\title{
Iron-associated protein interaction networks reveal the key functional modules related to survival and virulence of Pasteurella multocida
}

\begin{abstract}
Pasteurella multocida causes respiratory infectious diseases in a multitude of birds and mammals. A number of virulence-associated genes were reported across different strains of P. multocida, including those involved in the iron transport and metabolism. Comparative ironassociated genes of $\mathrm{P}$. multocida among different animal hosts towards their interaction networks have not been fully revealed. Therefore, this study aimed to identify the ironassociated genes from core- and pan-genomes of fourteen P. multocida strains and to construct iron-associated protein interaction networks using genome-scale network analysis which might be associated with the virulence. Results showed that these fourteen strains had 1587 genes in the core-genome and 3400 genes constituting their pan-genome. Out of these, 2651 genes associated with iron transport and metabolism were selected to construct the protein interaction networks and 361 genes were incorporated into the iron-associated protein interaction network (iPIN) consisting of nine different iron-associated functional modules. After comparing with the virulence factor database (VFDB), 21 virulence-associated proteins were determined and 11 of these belonged to the heme biosynthesis module. From this study, the core heme biosynthesis module and the core outer membrane hemoglobin receptor HgbA were proposed as candidate targets to design novel antibiotics and vaccines for preventing pasteurellosis across the serotypes or animal hosts for enhanced precision agriculture to ensure sustainability in food security.
\end{abstract}

Keyword: Comparative genomics; Functional modules related to virulence; Iron-associated genes; Pasteurella multocida; Protein interaction network 